This item was submitted to Loughborough's Research Repository by the author.

Items in Figshare are protected by copyright, with all rights reserved, unless otherwise indicated.

\title{
Hall-Lorenz number paradox in cuprate superconductors
}

PLEASE CITE THE PUBLISHED VERSION

LICENCE

CC BY-NC-ND 4.0

\section{REPOSITORY RECORD}

Alexandrov, A.S.. 2019. "Hall-lorenz Number Paradox in Cuprate Superconductors". figshare. https://hdl.handle.net/2134/1182. 


\title{
Hall-Lorenz number paradox in cuprate superconductors
}

\author{
A. S. Alexandrov \\ Department of Physics, Loughborough University, Loughborough, United Kingdom
}

\begin{abstract}
Significantly different normal state Lorenz numbers have been found in two independent direct measurements based on the Righi-Leduc effect, one about 6 times smaller and the other one about 2 times larger than the Sommerfeld value in single cuprate crystals of the same chemical composition. The controversy is resolved in the model where charge carriers are mobile lattice bipolarons and thermally activated nondegenerate polarons. The model numerically fits several longitudinal and transverse kinetic coefficients providing a unique explanation of a sharp maximum in the temperature dependence of the normal state Hall number in underdoped cuprates.
\end{abstract}

PACS numbers: 74.25.Fy,74.20.-z,72.15.Eb,74.72.-h

Particular interest in studies of high-temperature superconductors lies in a possible violation of the Wiedemann-Franz (WF) law in doped cuprates. A departure from the Fermi/BCS liquid picture is seen in both the superconducting and normal state thermal conductivities and might be related by a common mechanism 1 , 2]. Takenaka et al. [1] systematically studied the oxygencontent dependence of the insulating state thermal conductivity enabling them to estimate the phononic contribution, $\kappa_{p h}(T)$ for the metallic state to some extent. Their analysis led to the conclusion that the electronic term, $\kappa$ is only weakly $T$-dependent. This approximately $T$-independent $\kappa$ in the underdoped region therefore implies the violation of the WF law since the resistivity is found to be a non-linear function of temperature in this regime. A breakdown of the WF law has been seen in other cuprates such as $\mathrm{Pr}_{2-x} \mathrm{Ce}_{x} \mathrm{CuO}$ at very low temperatures 2]. On the other hand measurements by Proust et al. [3] on $\mathrm{Tl}_{2} \mathrm{Ba}_{2} \mathrm{Cu} 0_{6+\delta}$ have suggested that the Wiedemann-Franz law holds perfectly well in the overdoped region. However in any case the extraction of the electronic thermal conductivity has proven difficult and inconclusive as $\kappa$ and $\kappa_{p h}$ are comparable at elevated temperatures, or there is a thermal decoupling of phonons and electrons at ultra-low temperatures $\underline{4}$.

This uncertainty has been avoided in measurements of the Righi-Leduc effect. The effect describes transverse heat flow resulting from a perpendicular temperature gradient in an external magnetic field, which is a thermal analog of the Hall effect. Using the effect the "HallLorenz" electronic number, $L_{H}=\left(e / k_{B}\right)^{2} \kappa_{x y} /\left(T \sigma_{x y}\right)$ has been directly measured [5] in $\mathrm{YBa}_{2} \mathrm{Cu}_{3} \mathrm{O}_{6.95}$ and $\mathrm{YBa}_{2} \mathrm{Cu}_{3} \mathrm{O}_{6.6}$ since transverse thermal $\kappa_{x y}$ and electrical $\sigma_{x y}$ conductivities involve presumably only electrons. The experimental $L_{H}(T)$ showed a quasi-linear temperature dependence above the resistive $T_{c}$, which strongly violates the WF law. Remarkably, the measured value of $L_{H}$ just above $T_{c}$ turned out precisely the same as predicted by the bipolaron theory 6 , $L=0.15 L_{0}$, where $L_{0}=\pi^{2} / 3$ is the conventional Sommerfeld value. The breakdown of the WF law revealed in the Righi-Leduc effect [5] has been explained by a temperature-dependent contribution of thermally excited single polarons to the transverse magneto-transport [7].

Surprisingly more recent measurements of the HallLorenz number in single crystals of optimally doped $\mathrm{YBa}_{2} \mathrm{Cu}_{3} \mathrm{O}_{6.95}$ and optimally doped and underdoped $\mathrm{EuBa}_{2} \mathrm{Cu}_{3} \mathrm{O}_{y}$ led to an opposite conclusion [8]. The experimental $L_{H}$ for these samples has turned out only weakly temperature dependent and exceeding the Sommerfeld value by more than 2 times in the whole temperature range from $T_{c}$ up to the room temperature. Following an earlier claim [9] Matusiak and Wolf [8] have argued that a possible reason for such significant difference might be that Zhang et al. [5] used different samples, one for $\kappa_{x y}$ and another one for $\sigma_{x y}$ measurements, which makes their results for $L_{H}$ inconsistent.

Here I argue that there is no inconsistency in both $L_{H}$ determinations. One order of magnitude difference in two independent direct measurements of the normalstate Hall-Lorenz number is consistently explained by the bipolaron theory [10]. The theory explains the huge difference in the Hall-Lorenz numbers by taking into account the difference between the in-plane resistivity of detwinned [5] and twinned [8] single crystals. The theory fits well the observed $L_{H}(T)$ s and explains a sharp Hall-number maximum [8] observed in the normal state of underdoped cuprates.

In the presence of the electric field $\mathbf{E}$, the temperature gradient $\boldsymbol{\nabla} T$ and a weak magnetic field $\mathbf{B} \| \mathbf{z} \perp \mathbf{E}$ and $\boldsymbol{\nabla} T$, the electrical currents in $x, y$ directions are given by

$$
\begin{array}{r}
j_{x}=a_{x x} \nabla_{x}(\mu-2 e \phi)+a_{x y} \nabla_{y}(\mu-2 e \phi) \\
+b_{x x} \nabla_{x} T+b_{x y} \nabla_{y} T, \\
j_{y}=a_{y y} \nabla_{y}(\mu-2 e \phi)+a_{y x} \nabla_{x}(\mu-2 e \phi) \\
+b_{y y} \nabla_{y} T+b_{y x} \nabla_{x} T,
\end{array}
$$

and the thermal currents are:

$$
\begin{array}{r}
w_{x}=c_{x x} \nabla_{x}(\mu-2 e \phi)+c_{x y} \nabla_{y}(\mu-2 e \phi) \\
+d_{x x} \nabla_{x} T+d_{x y} \nabla_{y} T \\
w_{y}=c_{y y} \nabla_{y}(\mu-2 e \phi)+c_{y x} \nabla_{x}(\mu-2 e \phi) \\
+d_{y y} \nabla_{y} T+d_{y x} \nabla_{x} T .
\end{array}
$$


Here $\mu$ and $\phi$ are the chemical and electric potentials.

Real phonons and (bi)polarons are well decoupled in the strong-coupling regime of the electron-phonon interaction [10] so the standard Boltzmann equation for the kinetics of renormalised carriers is applied. If we make use of the $\tau(E)$-approximation [11] the kinetic coefficients of bipolarons are found as [7] $\left(k_{B}=\hbar=c=1\right)$

$$
\begin{aligned}
a_{x x}^{b} & =a_{y y}^{b}=\frac{2 e n_{b}}{m_{b}}\left\langle\tau_{b}\right\rangle, \\
a_{y x}^{b} & =-a_{x y}^{b}=\frac{2 e g_{b} B n_{b}}{m_{b}}\left\langle\tau_{b}^{2}\right\rangle, \\
b_{x x}^{b} & =b_{y y}^{b}=\frac{2 e n_{b}}{T m_{b}}\left\langle(E-\mu) \tau_{b}\right\rangle, \\
b_{y x}^{b} & =-b_{x y}^{b}=\frac{2 e g_{b} B n_{b}}{T m_{b}}\left\langle(E-\mu) \tau_{b}^{2}\right\rangle,
\end{aligned}
$$

and

$$
\begin{aligned}
c_{x x}^{b} & =c_{y y}^{b}=\frac{n_{b}}{m_{b}}\left\langle(E+2 e \phi) \tau_{b}\right\rangle, \\
c_{y x}^{b} & =c_{x y}^{b} \frac{g_{b} B n_{b}}{m_{b}}\left\langle(E+2 e \phi) \tau_{b}^{2}\right\rangle, \\
d_{x x}^{b} & =d_{y y}^{b}=\frac{n_{b}}{T m_{b}}\left\langle(E+2 e \phi)(E-\mu) \tau_{b}\right\rangle, \\
d_{y x}^{b} & =-d_{x y}^{b}=\frac{g_{b} B n_{b}}{T m_{b}}\left\langle(E+2 e \phi)(E-\mu) \tau_{b}^{2}\right\rangle,
\end{aligned}
$$

where

$$
\langle Q(E)\rangle=\frac{\int_{0}^{\infty} d E Q(E) E D_{b}(E) \partial f_{b} / \partial E}{\int_{0}^{\infty} d E E D_{b}(E) \partial f_{b} / \partial E},
$$

$D_{b}(E) \propto E^{d / 2-1}$ is the density of states of a $d$ dimensional bipolaron spectrum, $E=K^{2} /\left(2 m_{b}\right), g_{b}=$ $2 e / m_{b}$, and $f_{b}(E)$ is the equilibrium distribution function. Polaronic coefficients are obtained by replacing super/subscripts $b$ for $p$, double elementary charge $2 e$ for $e$ and $\mu$ for $\mu / 2$ in all kinetic coefficients, and $m_{b}$ for $2 m_{p}$ in $a_{i j}$ and $c_{i j}$. The kinetic energy of bipolarons, $E$ should be replaced by $E+T^{*}$, where $E=k^{2} /\left(2 m_{p}\right)$ is the polaron kinetic energy, and $T^{*}$ is half of the bipolaron binding energy (i.e. the pseudogap temperature in the theory 10 ).

The in-plane resistivity, $\rho$, the Hall number, $R_{H}$, and the Hall-Lorenz number, $L_{H}$ are expressed in terms of the kinetic coefficients as $\rho^{-1}=2 e a_{x x}, R_{H}=$ $a_{y x} / 2 e B\left(a_{x x}\right)^{2}$, and

$$
L_{H}=\frac{e\left[\left(d_{y x} a_{x x}-c_{y x} b_{x x}\right) a_{x x}-c_{x x}\left(b_{x x} a_{y x}-b_{y x} a_{x x}\right)\right]}{2 T a_{y x} a_{x x}^{2}},
$$

respectively, where $a, b, c, d=a^{p}+a^{b}, b^{p}+b^{b}, c^{p}+c^{b}, d^{p}+$ $d^{b}$.

The in-plane resistivity, the temperature-dependent paramagnetic susceptibility, and the Hall ratio have already been described by the bipolaron model taking into account thermally activated single polarons 12,13 ,
14, 15. The bipolaron model has also offered a simple explanation of $c$-axis transport and the anisotropy of cuprates 14, 16, 17, 18]. The crucial point is that single polarons dominate in $c$-axis transport at finite temperatures because they are much lighter than bipolarons in $c$-direction. Bipolarons can propagate across the planes only via a simultaneous two-particle tunnelling, which is much less probable than a single polaron tunnelling. However, along the planes polarons and inter-site bipolarons propagate with comparable effective masses [10]. Hence in the mixture of nondegenerate quasitwo-dimensional (2D) bosons and thermally excited 3D fermions, only fermions contribute to $c$-axis transport, if the temperature is not very low, which leads to the thermally activated $c$-axis transport and to the huge anisotropy of cuprates 16 .

We have also shown [7] that by the necessary inclusion of thermally activated polarons, the model, Eq.(4) predicts a breakdown of the WF law with the small nearlinear in temperature Hall-Lorenz number, as observed experimentally by Zhang et al. [5] (see Fig.1). Let us now show that the bipolaron model describes the contrasting observations of Ref. [8] as well, if the ratio of bipolaron and polaron mobilities, $\alpha=2 \tau_{b} m_{p} / \tau_{p} m_{b}$ becomes relatively small.

Both polaronic and bipolaronic carriers are not degenerate above $T_{c}$, so the classical distribution functions, $f_{b}=y \exp (-E / T)$ and $f_{p}=y^{1 / 2} \exp \left[-\left(E+T^{*}\right) / T\right]$ are applied with $y=\exp (\mu / T)$. The chemical potential is evaluated using $2 n_{b}+n_{p}=x / v_{0}$, where $x$ is the number of itinerant holes in the unit cell volume $v_{0}$ not localised by disorder. The bipolaron density remains large compared with the polaron density in a wide temperature range, so that $n_{b} v_{0} \approx x / 2$ and $y \approx \pi x /\left(m_{b} a^{2} T\right)$ for quasi-2D bipolarons. Then the atomic density of $3 \mathrm{D}$ polarons is $n_{p} v_{0}=$ $T m_{p} a^{2} \exp \left(-T^{*} / T\right)\left(x m_{p} / 2 \pi^{2} m_{b}\right)^{1 / 2} \quad(a$ is the lattice constant). The ratio $\beta=n_{p} / 2 n_{b}$ remains small at any pseudogap temperature $T^{*}$ and any relevant doping level $x>0.05, \beta \approx T \exp \left(-T^{*} / T\right)\left(18 m_{p} / \pi^{2} x m_{b}\right)^{1 / 2} / W \ll 1$, if the temperature $T$ is small compared with the polaron bandwidth $W=6 / m_{p} a^{2}$. Hence, if the mobility ratio $\alpha$ is of the order of unity, both longitudinal and transverse in-plane magnetotransport is dominated by bipolarons, which explains a remarkably low $L_{H}$ in high-quality detwinned crystals used in Ref. [5], Fig.1.

On the other hand, twinned crystals used in Ref. [8] had the in-plane resistivity several times larger than those of Ref. [5] presumably resulting from twin boundaries and long term aging. The twin boundaries and other defects are strong scatterers for slow 2D bipolarons (see below), while lighter quasi-3D polarons are mainly scattered by real optical phonons, which are similar in all crystals. Hence one can expect that $\alpha$ becomes small in twinned crystals of Ref. [8]. If the condition $\alpha^{2} \ll \beta$ is met, then only polarons contribute to the transverse electric and thermal magnetotransport. It explains about the same thermal Hall conductivities $\left(\kappa_{x y} \approx 2.5 \times 10^{-3} B \mathrm{~W} / \mathrm{Km}\right.$ at $\mathrm{T}=100 \mathrm{~K}$ ) dominated by polarons in both crystals of 


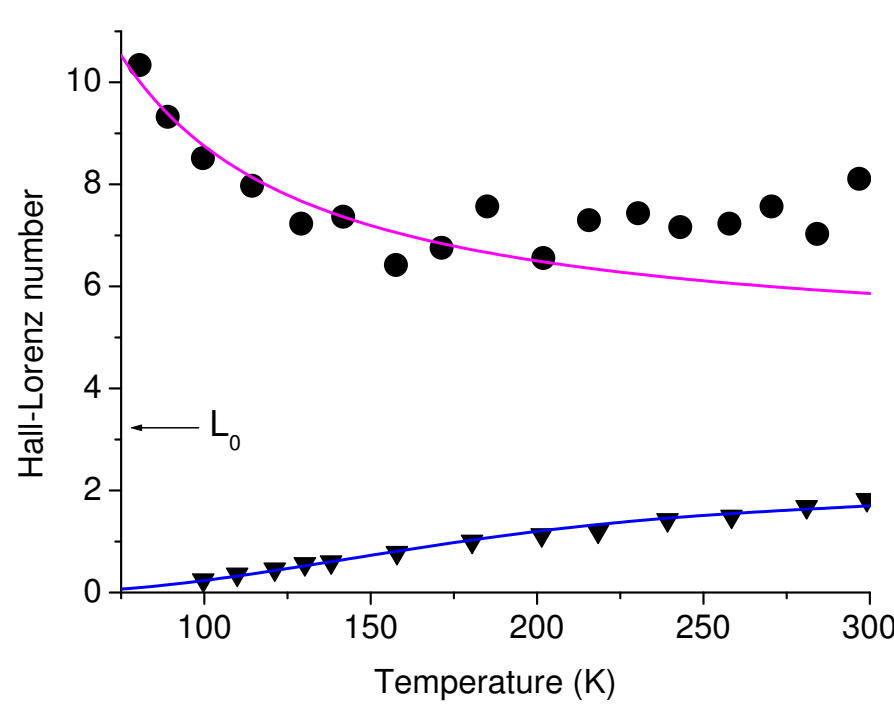

FIG. 1: The Hall-Lorenz number $L_{H}$ in underdoped twinned $\mathrm{EuBa}_{2} \mathrm{Cu}_{3} \mathrm{O}_{6.65}$ (circles) [8] compared with the theory, Eq.(7) when $\alpha \ll 1$ (upper line), and the significantly different HallLorenz number in detwinned $\mathrm{YBa}_{2} \mathrm{Cu}_{3} \mathrm{O}_{6.95}$ (triangles) [5] described by the same theory [7] with a moderate value of $\alpha=0.44$ (lower line).

$\mathrm{YBa}_{2} \mathrm{Cu}_{3} \mathrm{O}_{6.95}$ used in Ref. [5] and in Ref. [8], and at the same time a substantial difference of their electrical Hall conductivities, $\sigma_{x y}$, as bipolarons virtually do not contribute to $\sigma_{x y}$ in the twinned samples.

To arrive at simple analytical results and illustrate their quantitative agreement with the experiment [8] let us assume that $\alpha^{2} \ll \beta$, but $\alpha \gtrsim \beta$, and neglect an energy dependence of the transport relaxation rates of all carriers. In such conditions bipolarons do not contribute to transverse heat and electric flows, but determine the in-plane conductivity. Kinetic responses are grossly simplified as

$$
\begin{array}{r}
\rho=\frac{m_{b} v_{0}}{2 e^{2} x \tau_{b}} \\
R_{H}=\frac{v_{0} \beta}{e x \alpha^{2}}=\frac{e^{3} n_{p} \tau_{p}^{2}}{m_{p}^{2}} \rho^{2}, \\
L_{H}=4.75+3 T^{*} / T+\left(T^{*} / T\right)^{2} .
\end{array}
$$

As in the case of $\alpha^{2} \gtrsim \beta$, discussed in Ref. [7], the recombination of a pair of polarons into bipolaronic bound states at the cold end of the sample results in the breakdown of the WF law, as described by two temperaturedependent terms in Eq.(7). The breakdown is reminiscent of the one in conventional semiconductors caused by the recombination of electron-hole pairs at the cold end 11]. However, the temperature dependence and the value of $L_{H}(T)$ turn out remarkably different. When $\alpha^{2} \ll \beta$, The Hall-Lorenz number is more than by an order of magnitude larger than in the opposite regime, $\alpha^{2} \gtrsim$ $\beta$. It increases with temperature lowering rather than decreases fitting well the experimental observation $[8]$ in twinned underdoped single crystals of $\mathrm{EuBa}_{2} \mathrm{Cu}_{3} \mathrm{O}_{6.65}$ with $T^{*}=100 \mathrm{~K}$, Fig.1. Hence by varying the bipolaron to polaron mobility ratio, $\alpha$, the model accounts for qualitatively different behaviours of $L_{H}(T)$ in twinned and detwinned cuprates. The energy dependence of relaxation rates might somewhat change numerical coefficients in Eq.(7), but it does not qualitatively change the temperature dependence and the value of $L_{H}(T)$.

The Hall-Lorenz number is the ratio of different kinetic coefficients rather than a proper kinetic response function. However, its significant departure from the Sommerfeld value $L_{0} \approx 3.3$ clearly indicates a non-Fermi liquid behaviour since the relaxation mechanism virtually cancels in the ratio. The partially gapped Fermiliquid model used to explain large $L_{H}$ in Ref. [8] predicts a quadratic decrease of $L_{H}(T)$ with temperature lowering, rather than a steep increase as observed, Fig.1. To account for an unexpected rise of $L_{H}(T)$ below $T \approx 160 \mathrm{~K}$ in underdoped samples, Matusiak et al. [8] suggested an opening of another narrower gap. However the gapped Fermi liquid model is clearly incompatible with the near temperature-independent resistivity and with the sharp maximum of the normal state Hall ratio at $100 \mathrm{~K}$, as measured in Ref. [8], Fig.2. It is also hard to accept the claim of Refs. [8, [9] that the research team of Ref. [5] could so badly manipulate their data to arrive at an erroneous $L_{H}$ more than one order of magnitude smaller in identical cuprates.

On the contrary our model explains the near temperature-independent resistivity and the unusual Hall ratio, Fig.2. If we assume that in $\mathrm{EuBa}_{2} \mathrm{Cu}_{3} \mathrm{O}_{6.65}$ slow bipolarons are mainly scattered by neutral defects and twin boundaries, their relaxation rate depends on the temperature as $\tau_{b 0} / \tau_{b}=1+\left(T / T_{0}\right)^{1 / 2}$, where $\tau_{b 0}$ is a constant. The temperature independent contribution comes from the scattering rate off neutral impurities with the carrier exchange 11] similar to the scattering of slow electrons by hydrogen atoms. The square-root term originates in the scattering of slow bipolarons by point defects and twin boundaries with a temperature independent mean-free pass. The scale $T_{0}$ thus depends on the relative strength of two scattering mechanisms. The theoretical resistivity

$$
\frac{\rho(T)}{\rho_{0}}=1+\left(T / T_{0}\right)^{1 / 2}
$$

fits well the experimental $\rho(T)$ in the entire normalstate region with $\rho_{0}=m_{b} v_{0} /\left(2 e^{2} x \tau_{b 0}\right)=1.3 \times$ $10^{-5} \Omega \mathrm{m}$ and $T_{0}=321 \mathrm{~K}$, Fig. 2 . Lighter $3 \mathrm{D}$ polarons are scattered by defects and optical phonons, so that $\tau_{p 0} / \tau_{p}=(T / W)^{1 / 2}+B \exp (-\omega / T)$ with a temperatureindependent $\tau_{p 0}$. Then, using $n_{p} \propto T \exp \left(-T^{*} / T\right)$, Eq.(6) yields

$$
R_{H}(T)=\rho^{2}(T) \frac{A T \exp \left(-T^{*} / T\right)}{\left[T^{1 / 2}+b \exp (-\omega / T)\right]^{2}} .
$$

This expression fits extremely well the experimental 


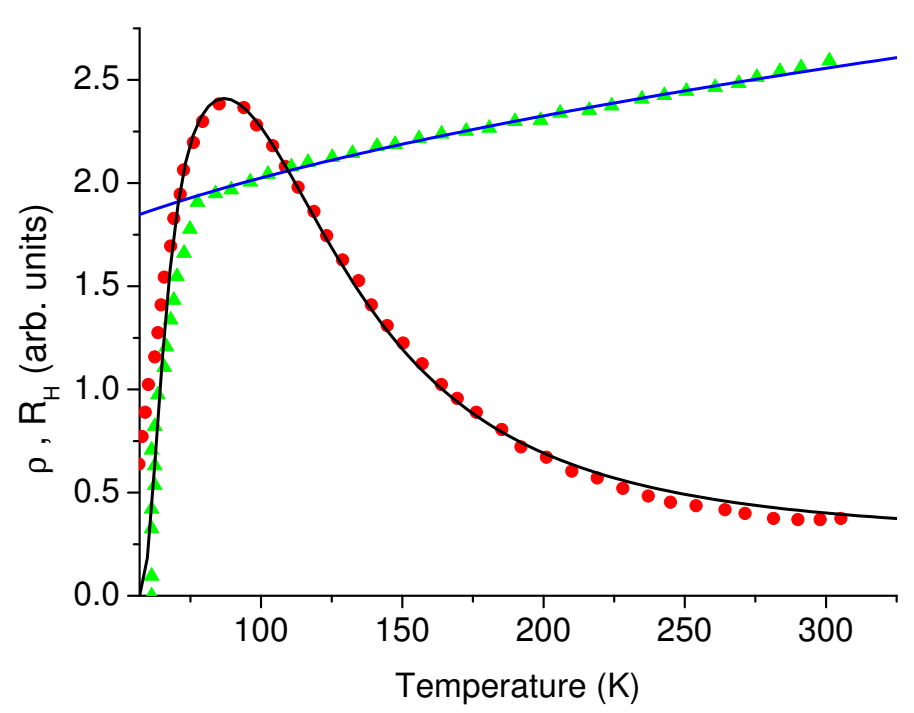

FIG. 2: The in-plane resistivity $\rho$ (triangles) and the Hall ratio $R_{H}$ (circles) of underdoped twinned $E u B a_{2} \mathrm{Cu}_{3} \mathrm{O}_{6.65}$, Ref. [8], compared with the theory (lines).

$R_{H}(T)$ with temperature independent constants $A=$ $e^{3} \tau_{p 0}^{2}\left(18 x m_{p} / \pi^{2} m_{b}\right)^{1 / 2} /\left(v_{0} m_{p}^{2}\right)=275 \mathrm{~m} / \Omega^{2} \mathrm{C}$ and $b=$ $B W^{1 / 2}=122 \mathrm{~K}^{1 / 2}$, the reasonable value of the characteristic optical phonon frequency $\omega=470 \mathrm{~K}$, and the same pseudogap $T^{*}=100 \mathrm{~K}$ as in the Hall-Lorenz number in Fig.1. It appears almost perfect even in the critical region very close to $T_{c}$, Fig.2, if one uses the experimental $\rho(T)$ in Eq.(9). However the maximum of the Hall ratio is a normal state feature lying well above the critical re- gion by about $30 \mathrm{~K}$, Fig.2, as in underdoped $\mathrm{YBa}_{2} \mathrm{Cu}_{3} \mathrm{O}_{y}$, Ref. [15]. At temperatures below $T^{*}$ the Hall ratio drops as the number of thermally activated polarons decreases, and at temperatures above $T^{*}$ it drops since the polaron relaxation time decreases.

To verify the self-consistency of the model let us estimate $\alpha$ and $\beta$. In the optimally doped samples one expects $\alpha^{2}$ of the order of $\beta$, so the Hall ratio approximately measures the itinerant carrier density, $R_{H}^{o p t} \approx v_{0} / e x_{o p t}$. Then using the experimental values [8] of $R_{H}$ in optimally doped $\mathrm{EuBa}_{2} \mathrm{Cu}_{3} \mathrm{O}_{7}$ and underdoped $\mathrm{EuBa} \mathrm{C}_{2} \mathrm{Cu}_{3} \mathrm{O}_{6.65}$ one estimates $\alpha^{2} / \beta \approx R_{H}^{o p t} x_{\text {opt }} / x R_{H} \lesssim 0.1$ in underdoped $\mathrm{EuBa}_{2} \mathrm{Cu}_{3} \mathrm{O}_{6.65}$, which justifies one of our assumptions. To get $\alpha \gtrsim \beta$ we have to assume that $\beta \lesssim 0.1$, which is indeed the case in the whole temperature range, if the polaron band is wide enough, $W \gtrsim 5000 \mathrm{~K}$. Finally using the values of $\rho_{0}$ and $A$ and taking $x=0.1$, $m_{p}=5 m_{e}, v_{0}=0.2 \mathrm{~nm}^{3}$ and $m_{b}=2 m_{p}$ the polaron and bipolaron mean-free pass is estimated as $l_{p} \approx 4 \mathrm{~nm}$ and $l_{b} \approx 0.3\left(m_{b} / m_{e}\right)^{1 / 2} \mathrm{~nm}$, respectively (here $m_{e}$ is the free electron mass). Their values are large compared with the lattice constant justifying the Boltzmann approximation for all carriers.

To sum up, the bipolaron theory resolves the paradox of very different Hall-Lorenz numbers found in two independent measurements [5, 8] in cuprate single crystals. It explains a flat temperature dependence of the in-plane resistivity and the sharp maximum in the normal-state Hall number of underdoped cuprates as well.

The author acknowledges support of this work by EPSRC (UK) (grant EP/C518365/1) and enlightening discussions with Nigel Hussey of thermal conductivity measurements.
[1] K. Takenaka, Y. Fukuzumi, K. Mizuhashi, S. Uchida, H. Asaoka and H. Takei, Phys. Rev. B56, 5654 (1997).

[2] R.W. Hill, C. Proust, L. Taillefer, P. Fournier and R.L. Greene, Nature, 414, 711 (2001).

[3] C. Proust, E. Boaknin, R.W. Hill, L. Taillefer and A.P. Mackenzie, Phys. Rev. Lett. 89, 147003 (2002).

[4] M.F. Smith, J. Paglione, M.B. Walker, and L. Taillefer, Phys. Rev. B71, 014506 (2005).

[5] Y. Zhang, N.P. Ong, Z.A. Xu, K. Krishana, R. Gagnon and L. Taillefer, Phys. Rev. Lett. 84, 2219 (2000), and unpublished.

[6] A.S. Alexandrov and N.F. Mott, Phys. Rev. Lett. 71, 1075 (1993).

[7] K.K. Lee, A.S. Alexandrov, and W.Y. Liang, Phys. Rev. Lett. 90, 217001 (2003); Eur. Phys. J. B30, 459 (2004).

[8] M. Matusiak and Th. Wolf, Phys. Rev. B72, 054508(R) (2005); M. Matusiak, K. Rogacki, T. Plackowski, and B. Veal, cond-mat/0412175 (2004).

[9] M.R. Li, Phys. Rev. B65, 184515 (2002).

[10] A.S. Alexandrov, Theory of Superconductivity: From
Weak to Strong Coupling (IoP Publishing, Bristol and Philadelphia, 2003).

[11] A. Anselm, Introduction of Semiconductor Theory (Prentice and Hall, New Jersey, 1981).

[12] A.S. Alexandrov, A.M. Bratkovsky, and N.F. Mott, Phys. Rev. Lett. 72, 1734 (1994).

[13] W.M. Chen, J.P. Franck, and J. Jung, Physica C341, 1875 (2000).

[14] X.H. Chen, M. Yu, K.Q. Ruan, S.Y. Li, Z. Gui, G.C. Zhang, and L.Z. Cao, Phys. Rev. B58, 14219 (1998).

[15] A.S. Alexandrov, V.N. Zavaritsky, and S. Dzhumanov, Phys. Rev. B69, 052505 (2004).

[16] A.S. Alexandrov, V.V. Kabanov, and N.F. Mott, Phys. Rev. Lett. 77, 4796 (1996).

[17] J. Hofer, J. Karpinski, M. Willemin, G.I. Meijer, E.M. Kopnin, R. Molinski, H. Schwer, C. Rossel, and H. Keller, Physica C 297, 103 (1998).

[18] V.N. Zverev and D.V. Shovkun, JETP Lett. 72, 73 (2000). 